\title{
EVALUACIÓN Y DETERMINACIÓN DE LA PRODUCCIÓN DE VINO Y ALCOHOL A PARTIR DE EXUDADO DE CACAO, COOPERATIVA MULTIFUNCIONAL CACAOTERA LA CAMPESINA R.L, DEL MUNICIPIO DE MATIGUÁS EN EL DEPARTAMENTO DE MATAGALPA.
}

\section{EVALUATION AND DETERMINATION OF WINE AND ALCOHOL PRODUCTION FROM CACAO EXUDATE, MULTIFUNCTIONAL COOPERATIVE CACAOTERA LA CAMPESINA RL, OF THE MUNICIPALITY OF MATIGUÁS IN THE DEPARTMENT OF MATAGALPA}

Alba Veranay Díaz Corrales ${ }^{1}$ Yessin Mahela Calero Leiva² Zilgean Massiel Moreno Lazo ${ }^{3}$

El presente estudio tuvo como propósito la "Evaluación y determinación de la producción de vino y alcohol a partir de exudado de cacao de la mezcla de variedades forastero amazónico, criollo e hibrido a escala de laboratorio, así como sus costos de producción, para la Cooperativa Multifuncional Cacaotera La Campesina R.L, del municipio de Matiguás en el departamento de Matagalpa". En el desarrollo de esta investigación se realizaron visitas a la cooperativa para recolección del mucilago o exudado, el exudado recolectado se trasladó al laboratorio de Agroindustria de la Universidad Nacional de Ingeniería, Sede Estelí (UNIRUACS), para ser procesado utilizando dos tratamientos uno referente a la pasteurización del exudado (T1) y otro a la no pasteurización del exudado (T2). En el vino se obtuvieron rendimientos de $69.33 \%$ para $\mathrm{T} 1$ y $70.31 \%$ para $\mathrm{T} 2$, siendo estos mayores al $60 \%$, con un grado de alcohol $13.23 \%$ en $\mathrm{T} 1$ y $12.61 \%$ en T2, clasificándose en un vino seco. En relación al alcohol etílico obtenido al 75\%, fue un rendimiento de $58.4 \%$ para T1 y $76.09 \%$ para T2. Los costos de producción se calcularon en base a rendimientos obtenido en cada uno de los proceso siendo para el vino con T1 de C\$198.12 córdobas y para vino de T2 C\$195.23 córdobas, para el alcohol con tratamiento de pasteurización C $\$ 157.58$ y alcohol del tratamiento no pasteurizado de $\mathrm{C} \$ 124.47$; según resultados de este estudio si se pueden obtener el vino y el alcohol a partir del exudado de cacao con características comerciales, obteniendo mejores rendimientos en producción de vino que en el alcohol.

PALABRAS CLAVE: mucilago; cacao; alcohol; vino

ABSTRACT: The purpose of this research was: To evaluate and to determine the production of wine and alcohol from the cocoa exudate of the blend of Amazonian, Creole and Hybrid varieties at laboratory scale, as well as their production costs, for the Cooperative Multifuncional Cacaotera La Campesina RL, of the municipality of Matiguás in the department of Matagalpa ". For the development of this research, visits were made to the cooperative to collect the mucilage

\footnotetext{
${ }^{1}$ Coordinadora de Investigación UNI-RUACS, Nicaragua alba.diaz@norte.ni.edu.ni - Autor para la correspondencia.

${ }^{2}$ Graduada Ingeniería Agroindustrial UNI-RUACS, Nicaragua

${ }^{3}$ Graduada Ingeniería Agroindustrial UNI-RUACS, Nicaragua
} 
or exudate. The collected exudate was transferred to the Laboratory of Agroindustry of the National University of Engineering, Estelí Headquarters (UNI-RUACS), to be processed using two treatments, the first Referring to the pasteurization of the exudate (T1), and the other one to the non-pasteurization of the exudate (T2). In the wine, were obtained yields of: $69.33 \%$ for T1 and $70.31 \%$ for $\mathrm{T} 2$, being these greater than $60 \%$, with alcohol degrees of $13.23 \%$ in $\mathrm{T} 1$ and $12.61 \%$ in $\mathrm{T} 2$, being classified as a dry wine. In relation to ethyl alcohol obtained at $75 \%$, it was a yield of $58.4 \%$ for $\mathrm{T} 1$ and $76.09 \%$ for $\mathrm{T} 2$. The costs of production were calculated based on yields obtained in each of the processes being for wine with T1 of C\$198.12 Córdobas and for wine of T2 C\$ 195.23 Córdobas, for alcohol with pasteurization treatment C\$ 157.58 and treatment alcohol non-Pasteurized from C\$ 124.47; According to the results of this research, wine and alcohol can be obtained from the exudate of cacao with commercial characteristics, getting better yields in wine production than in alcohol.

KEYWORDS: mucilage; cocoa; alcohol; wine

\section{INTRODUCCIÓN}

El cacao se cultiva por sus granos, los cuales se hallan contenidos en grandes mazorcas rojas o amarillas que nacen directamente de los tallos y ramas del árbol, cada mazorca contiene, aproximadamente, una tercera parte de su peso de granos encontrados en un mucílago blanco, el mucílago y los granos se sacan de la mazorca y se fermentan. Durante el procesamiento del cacao se generan residuos provenientes de la fermentación. De 800 kilos de semilla fresca de cacao se obtiene 40 litros de desecho, como es el mucílago o exudado como comúnmente se le conoce, (Garzaro, Guerra Cedezo, \& Kalvatchev, 1998).

En el año 2004, en la revista de comercio exterior se indica que el cultivo del cacao es nativo de nuestro país, siendo cultivado en las zonas tropicales de Nicaragua, como es la región del Atlántico Sur (Nueva Guinea, Bluefields, Kukra Hill), la zona de Las Minas (Siuna, Bonanza y Rositas) y el Atlántico Norte (Waslala, Río Coco), Matagalpa, Jinotega y Rivas.

La Cooperativa Multifuncional Cacaotera La Campesina cuenta con el apoyo 270 productores socios activos y 180 tramitando su inclusión con un área total de 1300 manzanas de cacao, de las comarcas pertenecientes a los municipios Matiguás, Río Blanco y Muy Muy (departamento de Matagalpa) y en Bocana de Paiwas (Región Autónoma Atlántico Sur), (Castellón, 2012).

Actualmente al exudado obtenido del proceso de beneficiado de cacao realizado por la cooperativa, no se les da tratamiento a este residuo, siendo solamente desechado, por lo tanto generando contaminación al medio ambiente, afectando la salud de las personas aledañas al lugar, a los animales existentes provocando intoxicaciones y una mala imagen.

Por lo anterior el presente estudio "Evaluación y determinación de la producción de vino y alcohol a partir de exudado de cacao de la mezcla de variedades forastero amazónico, criollo e hibrido a escala de laboratorio, así como el cálculo de los costos de producción", tuvo como objetivo el aprovechamiento del exudado para la obtención de productos. Este estudio se 
realizó con materia prima proveniente de la cooperativa Multifuncional Cacaotera La Campesina R.L., la cual se dedica al procesamiento del cacao.

Siendo esta investigación muy importante para presentar a los productores de la cooperativa La Campesina R.L, una alternativa para dar uso a este residuo y con su posterior aplicación aprovecharlo para la elaboración de vino y alcohol. En el estudio se realizaron visitas de campo, entrevistas, fase experimental, análisis bromatológicos y pruebas de catación para determinar la calidad y aceptabilidad de los productos obtenidos por parte de los consumidores.

\section{METODOLOGÍA}

La investigación se realizó a escala de laboratorio en las instalaciones del laboratorio de Agroindustria de la Universidad Nacional de Ingeniería Sede regional del Norte, Recinto Universitario Augusto C. Sandino (UNI-RUACS), ubicada en Estelí. Utilizando como materia prima (exudado de Cacao) proveniente de la Cooperativa La Campesina R.L.

Siendo la investigación realizada del tipo experimental, ya que se obtuvo alcohol y vino a partir del exudado de cacao, mediante la aplicación del diseño experimental completamente aleatorio. Se determinaron las unidades experimentales y repeticiones necesarias para cada tratamiento, donde se analizó el efecto de la variable pasteurización, aplicando el diseño experimental completamente aleatorio, en dos tratamientos, uno haciendo la pasteurización del exudado de cacao y el otro la no pasteurización del exudado (testigo). También de cada unidad experimental en ambos tratamientos, se realizó un total de 15 muestras de 1 litro, para el análisis de variabilidad en los datos de $\mathrm{pH}$, grados Brix y rendimiento.

Las formulaciones del vino y el alcohol se realizaron tomando como base dos tratamientos para la elaboración del vino y dos para la elaboración del alcohol, divididos los tratamientos en la pasteurización y no pasteurización de la materia prima, utilizando una mezcla de exudado proveniente de las variedades de cacao forastero amazónico, híbrido y criollo.

Tabla 1: Tratamientos aplicados en la elaboración del vino y alcohol a partir de exudado de cacao

\begin{tabular}{cc|} 
Simbología & Tlpo de Tratamiento \\
\hline T1: & Mezcla de exudado de cacao pasteurizado \\
\hline T2: & Mezcla de exudado de cacao no pasteurizado \\
\hline
\end{tabular}

Fuente: Elaboración propia

Para obtener la materia prima se realizó visitas a la cooperativa de Cacao La Campesina R.L, ubicada en el municipio de Matiguás, para presentar la investigación y los beneficios que se obtendrían de ella, consiguiendo de esta manera la colaboración del gerente y los productores asociados a esta cooperativa con respecto a la obtención de la materia prima lo que se hizo en visitas posteriores. La recepción de los 35 litros de exudado de cacao para la realización de ambos productos, se hizo utilizando plástico transparente con cabuya, ubicados debajo de los 
cajones de fermentación, para posteriormente ubicarlo en 4 recipientes plásticos, (ver Figura 1), con capacidad de 10 litros cada uno para posteriormente transportarlos al laboratorio de Agroindustria de la UNI-RUACS, Sede Estelí.

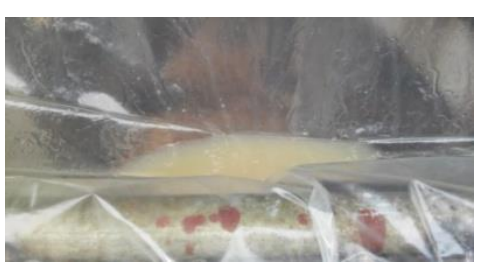

Figura 1: Recolección de materia prima

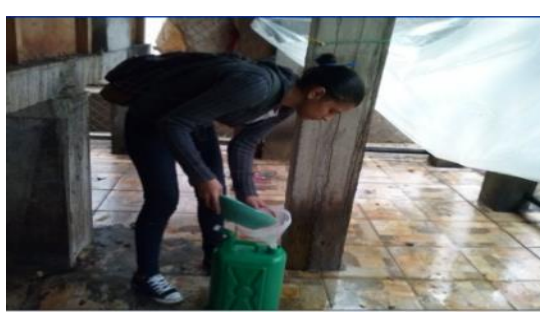

Figura 1: Llenado de recipientes plásticos

En el laboratorio de Agroindustria la materia prima se dividió como se muestra en la Figura 2:

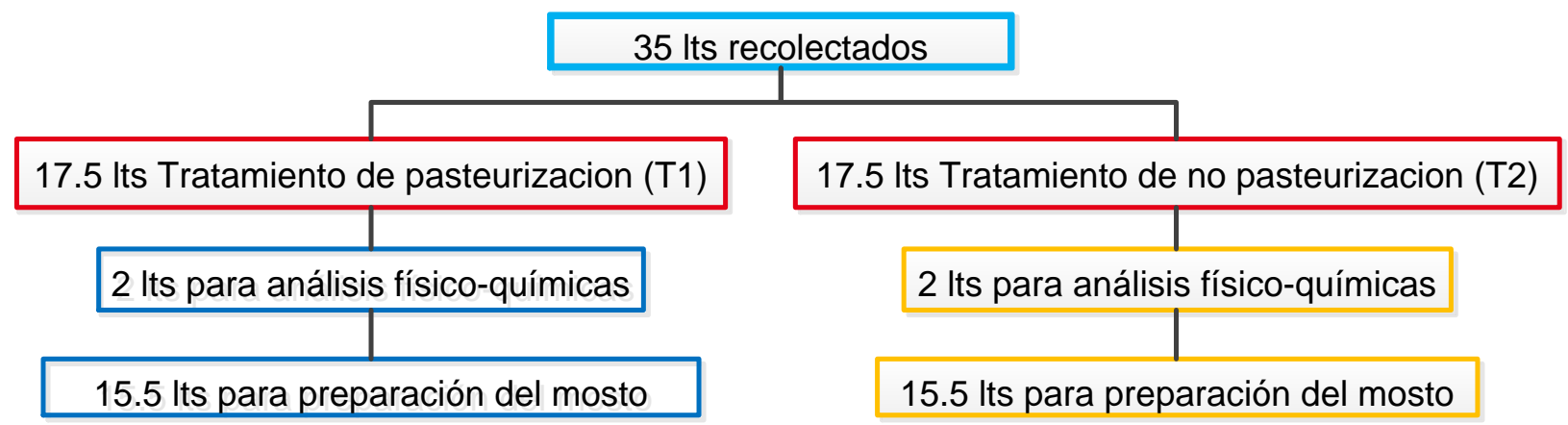

Figura 2: Distribución de exudado recolectado en la primera visita.

Se tomó una muestra de dos litros por tratamiento para análisis físico-químicos y se enviaron al LABAL-MIFIC, para realizar pruebas de acidez, cenizas, humedad, carbohidratos, proteína y fibra. En el laboratorio de agroindustria de la UNI Sede Regional Norte, ubicada en Estelí se realizó medición de $\mathrm{pH}$ y grados Brix. Posteriormente los 15 litros que quedaron para el tratamiento $\mathrm{T} 1$ se sometieron a filtración utilizando coladores, tinas plásticas y recipientes de aluminio, para posteriormente pasteurizar a temperatura de $75^{\circ} \mathrm{C}$ en una olla de aluminio y utilizando un termómetro, luego el exudado pasteurizado se dejó enfriar por 20 minutos hasta alcanzar una temperatura de $25^{\circ} \mathrm{C}$ a $30^{\circ} \mathrm{C}$, para adicionar la levadura Saccharomyces cerevisiae y que esta pudiera actuar al preparar el mosto en cada una de las 15 muestras. Cada muestra de 1 litro de exudado de cacao se preparó adicionando 0.126 Its de azúcar (200gr de azúcar) y 0.0080 lts de levadura (5 gr azúcar), las que se dejaron en fermentación por 15 días acompañando el mosto con una trampa de agua, conectados a través de una manguera permitiendo así la liberación del $\mathrm{CO}_{2}$ y evitar la entrada de oxígeno en las 15 muestras en estudio de T1.

Durante la fermentación cada 4 días se media a una de la muestras el pH con pH- neutro y los grados Brix con un refractómetro, terminada la fermentación se procedió a decantar dos veces 
pasando de un recipiente plástico a otro con objeto de separar la parte liquida de los sólidos originados durante la fermentación, para terminar de retirar sólidos en suspensión se filtró el vino obtenido, utilizando papel filtro y así obtener un producto más claro, el que posteriormente se envaso en botellas de vidrio a las que se les proporciono su respectiva etiqueta con un corcho y una capsula en la parte superior de la botella. Para el vino obtenido de T2 se siguieron las mismas operaciones y formulación antes descritas a excepción de la operación de pasteurización.

En relación a la obtención de alcohol a partir del exudado de cacao, se realizó una segunda recolección de la materia prima logrando recolectar solamente 10 litros de exudado, esto se debe a que la cosecha de cacao se encontraba en temporada baja por lo que la producción de exudado es menor, divididos los 10 litros como se muestra en la Figura 4:

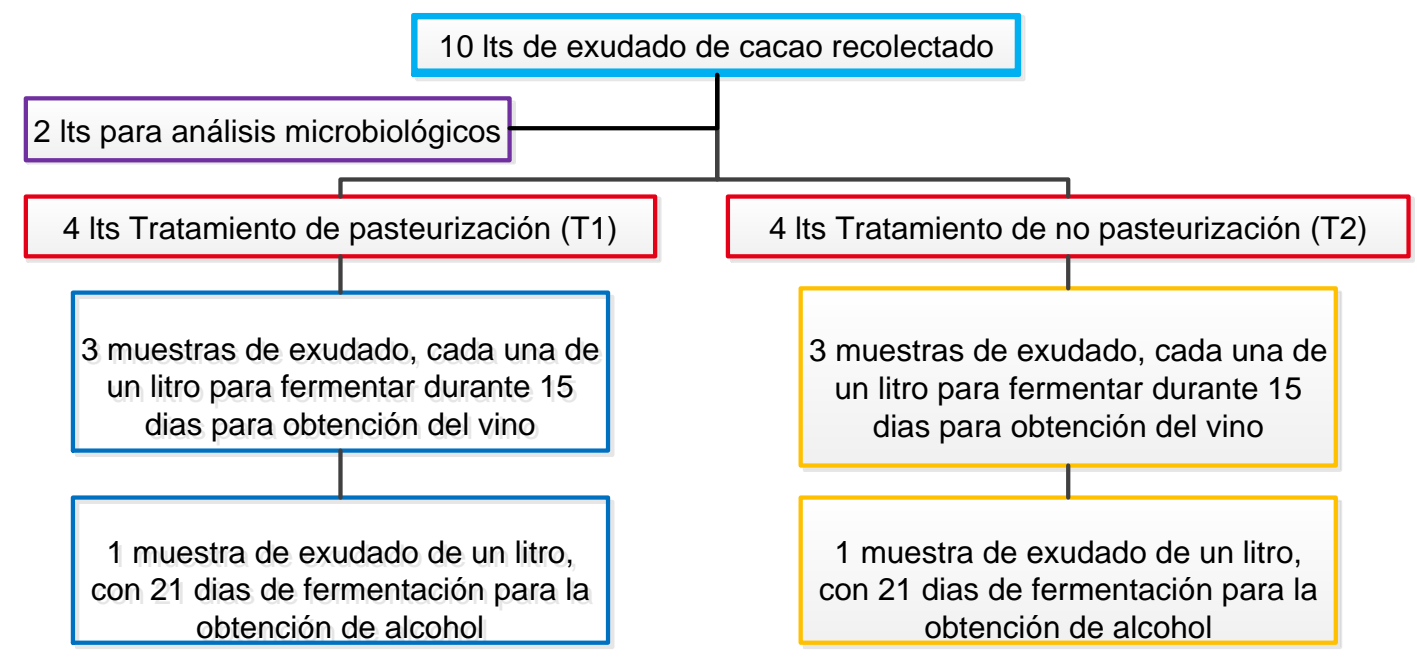

Figura 3: distribución de exudado recolectado en la segunda visita

Para obtener alcohol a partir del Tratamiento de Pasteurización (T1), se tomaron 5 muestras al azar del vino obtenido a partir de T1, las muestras tenían 30 días de estar en las botellas, antes de la etapa de destilación en el Laboratorio ISNAYA ubicado en la ciudad de Estelí. En esta destilación se utilizó un rota vapor a una temperatura de $75^{\circ} \mathrm{C}$, ver Figura número 5 . 


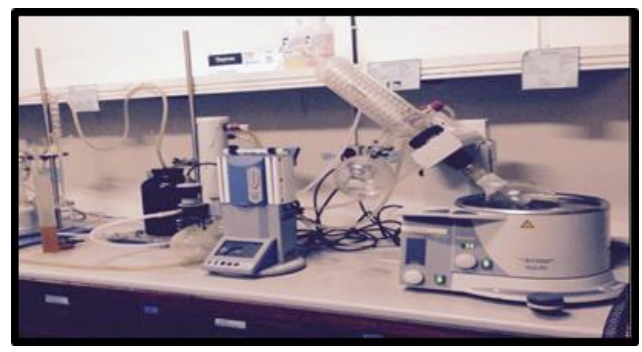

Rota vapor utilizado en la primera destilación

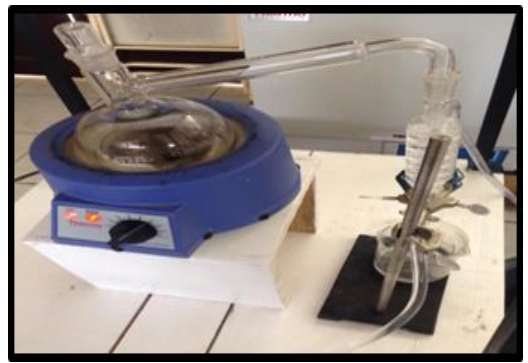

Destilador sencillo utilizado en segunda y tercera destilación

Figura 5: Equipos utilizados en la destilación

Con objeto de verificar si existían diferencias significativas en relación a los porcentaje de alcohol y rendimiento, con respecto a los días del vino en botella y días de fermentación del mosto, se preparó una muestra de 1lt de exudado de cacao obtenida a partir de la segunda recolección de la materia prima y se destiló pasados los 21 días de fermentación.

Para realizar el tratamiento correspondiente a la no pasteurización T2, también se tomaron 5 muestras al azar del vino obtenido, luego de haber pasado 30 días en botella. Las muestras se destilaron en el Laboratorio ISNAYA en un rota vapor a temperatura de $75^{\circ} \mathrm{C}$ al igual que en el tratamiento de pasteurización (T1). De igual manera que en T1 se tomó una muestra de la segunda recolección de exudado, para conocer el rendimiento por botella de $750 \mathrm{ml}$ y verificar si existían diferencias significativas entre los días en botella, días de fermentación del mosto y el porcentaje de alcohol obtenido, la destilación se realizó en el laboratorio de la UNI-RUACS.

\section{Calidad y aceptabilidad del vino:}

Para medir la calidad tanto del vino pasteurizado (T1) como del vino no pasteurizado (T2), se tomó una muestra de 2,000 ml (2lts), de cada una de ellas. Ambas muestras se enviaron en dos recipientes separados y codificados al laboratorio LABAL, para que se le realizaran análisis de grado alcohólico, acidez volátil y acidez total, así como porcentaje de metanol y azúcares reductores. En cuanto a $\mathrm{pH}$ y grados ${ }^{\circ}$ Brix, éstas se realizaron en el laboratorio de Agroindustria de la UNI-RUACS, tomando $200 \mathrm{ml}$ por cada una de las 15 muestras de los tratamientos en estudio, utilizando un $\mathrm{pH}$-metro y un refractómetro.

La aceptabilidad del vino para T1 y T2 se midió por medio de cataciones en las que participaron 67 panelistas mayores de 18 años, la cantidad de panelista fue obtenido mediante la aplicación de la fórmula de cálculo de poblaciones $n=\frac{Z^{2} * p * q * N}{\mathrm{~N} * e^{2+} Z^{2} * p * q}$ (Munch \& Angeles, 1996, pág. 103), considerando para ello una población de consumo de 3000 personas correspondiente al 3\% de la población económicamente activa (PEA) del municipio de Estelí que es de 99,760 habitantes correspondiente a un $79.5 \%$ del PEA total, (INIFOM, 2011). 
En donde:

$>Z=$ Nivel de confianza

$>\mathrm{N}=$ Universo, tamaño de la población

$>\mathrm{p}=$ probabilidades a favor es de 0.5

$>\mathrm{q}=$ probabilidades en contra, es de 0.5

$>\mathrm{e}=$ error de estimación

$>\mathrm{n}=$ tamaño de muestra

A cada catador participante de la comunidad educativa de la UNI-RUACS, Sede Estelí, se les entrego una ficha de catación por tratamiento para que pudieran evaluar los atributos organolépticos del vino procedente de T1 y T2, se les explico la metodología de llenado y la importancia de su colaboración. Las cataciones fueron realizadas en un lapso de 6 horas, ya que los panelista fueron pasando de cuatro en cuatro a la sala de catación.

\section{Calidad del Alcohol:}

Para realizar las pruebas de calidad del alcohol se preparó una muestra de $188 \mathrm{ml}$ del alcohol obtenido del tratamiento pasteurizado (T1) y $100 \mathrm{ml}$ del alcohol obtenido del tratamiento no pasteurizado (T2), para realizar los siguientes análisis:

$>$ Solubilidad: Se agregaron por medio de una bureta 4 gotas de alcohol y $1 \mathrm{ml}$ de disolvente (agua) en un tubo de ensayo, para cada uno de los tratamientos y luego se midió la acidez por medio de bandas de pH, (Sandoval, 2013).

Porcentaje de alcohol: Se tomaron $250 \mathrm{ml}$ en una probeta en la cual se introdujo el alcoholímetro y se procedió a la lectura del mismo.

$>$ pH: Se agregaron $100 \mathrm{ml}$ de alcohol en beaker, al que se le introdujo una banda de pH dejándola reposar cinco minutos en el alcohol y posteriormente hacer la lectura.

$>$ Densidad: Se colocó en una probeta $10 \mathrm{ml}$ de la sustancia a temperatura de $20^{\circ} \mathrm{C}$, los que posteriormente fueron pesados en una balanza analítica, para poder hacer la relación entre masa/volumen de la sustancia y haciendo uso de la fórmula de densidad poder conocer la densidad del alcohol obtenido.

$$
\rho=\frac{\text { masa }}{\text { volumen }}
$$

> Combustión: Se agregó $20 \mathrm{ml}$ de alcohol en una tapa al que después se le acercó un fósforo encendido, para que se diera el desprendimiento de una llama amarilla con azul no tan luminosa. 


\section{Costos de producción:}

Se tomó en cuenta los costos variables unitarios, para lo que fue necesario realizar cotizaciones de los costos en cuanto a materia prima, mano de obra directa y material directo utilizado, del mismo modo para establecer el salario de los trabajadores se tomó en cuenta lo estipulado por Briones, en el año 2015), en la Ley de Salario Mínimo, № 625.

Para los costos indirectos de fabricación se hizo en base al Reglamento de Equidad Fiscal, con reformas incorporadas, en el capítulo III de depreciación y determinación, articulo 63 de cuotas de depreciación y amortización, (Gueyer \& Montealegre, 2003), ademas se tomo en cuenta las prestaciones sociales de los trabajadores. Los costos variables unitarios y los costos indirectos de fabricación generados se digitaron en una hoja de cálculo de Excel para su posterior interpretación, los que permitieron obtener el costo de producir el vino y el alcohol obtenido por cada presentación, a través de la siguiente formula:

Ecuación 1: Cálculo de Costos Totales de Producción (CTP), donde CVU: costos variables unitarios y CF: Costos Fijos.

$$
\mathrm{CTP}=\mathrm{CVU}+\frac{\mathrm{CF}}{\text { Producción esperada }}
$$

\section{RESULTADOS Y DISCUSIÓN}

Con respecto a la caracterización físico-química los resultados obtenidos en el Laboratorio de Tecnologías de Alimentos LABAL-MIFIC, ubicados en Managua se describen en la Tabla 2:

Tabla 2: Caracterización físico-química de la materia prima

\begin{tabular}{|c|c|c|c|c|}
\hline \multicolumn{5}{|c|}{ Características físico-químicas de exudado de cacao no pasteurizado (T1) } \\
\hline $\begin{array}{l}\text { Descripción de } \\
\text { muestras }\end{array}$ & $\begin{array}{l}\text { Método de análisis } \\
\text { utilizado }\end{array}$ & Análisis & $\begin{array}{c}\text { Resultados de la } \\
\text { muestra }\end{array}$ & Unidades \\
\hline \multirow{8}{*}{ Exudado de cacao T1 } & \multirow{8}{*}{$\begin{array}{c}\text { OFFICIAL METHODS } \\
\text { OF ANALYSIS AOAC } \\
\text { Cap. 7-9-14 } \\
\text { Números: } 7.007-7.015- \\
\text { 7.056-7.070-9-021- } \\
\text { 9.062-14.006 }\end{array}$} & Humedad & 89,29 & $\%$ \\
\hline & & Proteína $(\mathrm{N} x$ 6,25) & 0,31 & $\%$ \\
\hline & & Grasa & 0 & $\%$ \\
\hline & & Ceniza & 0,49 & $\%$ \\
\hline & & Carbohidratos & 9,91 & $\%$ \\
\hline & & Fibra & 0 & $\%$ \\
\hline & & Alcohol $\left(20^{\circ} \mathrm{C}\right)$ & 4,38 & $\%$ \\
\hline & & Acidez (como Ac. Acético) & 0,11 & $\%$ \\
\hline \multicolumn{5}{|c|}{ Características físico-químicas de exudado de cacao no pasteurizado (T2) } \\
\hline $\begin{array}{l}\text { Descripción de } \\
\text { muestras }\end{array}$ & $\begin{array}{l}\text { Método de análisis } \\
\text { utilizado }\end{array}$ & Análisis & $\begin{array}{l}\text { Resultados de la } \\
\text { muestra }\end{array}$ & Unidades \\
\hline Exudado de cacao & OFFICIAL METHODS & Humedad & 94,10 & $\%$ \\
\hline
\end{tabular}


Alba V. Díaz Corrales; Yessin Mahela Calero Leiva; Zilgean Massiel Moreno Lazo

\begin{tabular}{|c|c|c|c|c|}
\hline \multirow[t]{7}{*}{ T2 } & \multirow{7}{*}{$\begin{array}{c}\text { OF ANALYSIS AOAC } \\
\text { Cap. 7-9-14 } \\
\text { Números: } 7.007-7.015- \\
7.056-7.070-9-021- \\
9.062-14.006\end{array}$} & Proteína $(\mathrm{N} \times 6,25)$ & 0,34 & $\%$ \\
\hline & & Grasa & 0 & $\%$ \\
\hline & & Ceniza & 0,53 & $\%$ \\
\hline & & Carbohidratos & 5,03 & $\%$ \\
\hline & & Fibra & 0 & $\%$ \\
\hline & & Alcohol $\left(20^{\circ} \mathrm{C}\right)$ & 4,02 & $\%$ \\
\hline & & Acidez (como Ac. Acético) & 0,13 & $\%$ \\
\hline
\end{tabular}

Fuente: (LABAL L. d., 2015)

Las variantes que se presentan en los resultados son correspondientes a los tratamientos $\mathrm{T} 1 \mathrm{y}$ T2, a los que se encontraban sometidas las muestras. Los resultados de ambas muestran manifiestan que si es posible procesar este residuo y obtener otros productos del mismo en este caso vino y alcohol, ya que los porcentajes de alcohol $(4.38 \%$ (T1) y $4.02 \%$ (T2)) y carbohidratos $(9.91 \%$ (T1) y $5.03 \%$ (T2)), encontrados tanto en el tratamiento pasteurizado (T1), como en el tratamiento no pasteurizado (T2), son bastantes considerables en relación a la fibra que posee $0 \%$ en ambas muestras de los tratamientos T1 y T2, lo que es satisfactorio al ser este componente (fibra) un inhibidor del azúcar necesaria para el buen desarrollo de la fermentación.

Con respecto a los análisis realizados en el laboratorio de Agroindustria de UNI-RUACS sede Estelí, se obtuvo un $\mathrm{pH}$ de 4, en ambos tratamiento, con grados ${ }^{\circ}$ Brix de 10.

En cuanto a los análisis microbiológicos para determinar la presencia de coliformes fecales y salmonella, los resultados obtenidos del Laboratorio de Tecnología de Alimentos (LABALMIFIC) se detallan en la Tabla 3:

Tabla 3: Resultado de análisis microbiológicos de exudado de cacao

Descripción de las muestras Método de Análisis Utilizado Análisis
Resultados de la muestra

Unidades

$\mathrm{NMP} / \mathrm{mL}$

\section{Exudado de cacao}

$$
\begin{aligned}
& \text { Método del Numero más } \\
& \text { Probable (NMP/mL). }
\end{aligned}
$$

\begin{tabular}{ccc} 
Coliformes fecales & $<3$ & $\mathrm{NMP} / \mathrm{mL}$ \\
\hline Salmonella & Ausencia & P-A/25mL
\end{tabular}

\section{Fuente: (LABAL L. d., 2015)}

Con respecto a coliformes fecales en la muestra de exudado de cacao enviada tiene una presencia en un rango de menos tres según el método del número más probable por mililitro $(<3$ $\mathrm{NMP} / \mathrm{mL}$ ), el cual representa un riesgo de tipo $B$ con una mediana probabilidad de causar daño a la salud, , según (MINECO, CONACYT, MIFIC, SIC, \& MEIC, 2009), por lo que se debe tener cuidado al momento de procesar esta materia prima, ya que las coliformes son bacterias que sobreviven en el ambiente debido a la presencia del oxígeno al pertenecer al género aeróbicas, cabe destacar que estas bacterias pueden llegarse a eliminar durante el proceso de fermentación al darse esta en un ambiente anaeróbico. En relación a la salmonella no se detectó presencia de esta en la materia prima (exudado de cacao), lo que favorece al producto realizado ya que no representaría un riesgo a la salud. 
En relación a determinar el proceso de obtención de vino y alcohol a partir de exudado de cacao:

$>$ En el vino no se encontraron diferencias significativas en cuanto a rendimientos y variables a medir como $\mathrm{pH}$ y grados Brix en los tratamientos aplicados, siendo para el tratamiento de pasterización (T1) los grados Brix se encontraron de 8 a 7.6, con un pH de 3.27 a 3.12, esto durante los 15 días de fermentación obteniendo un rendimiento de $69.33 \%$ equivalente a 10.4 Its de exudado de los 15 destinados a este tratamiento. En relación al tratamiento de no pasteurización (T2), los grados Brix variaron de 7.60 a 3.00 , con un $\mathrm{pH}$ de 3.21 a 3.12 y un rendimiento de $70.31 \%$ equivalente a 10.89 Its de los 15.5 Its destinados a este tratamiento. Ambos grados Brix están por debajo del rango establecido por Zeledón, Arvizú Aráuz, \& González Urrutia, en el año 2013, del mismo modo el pH se encuentra dentro del rango de 3.01 a 3.76 establecido por Clímaco Álvarez, en el año 2002.

$>$ En relacion al alcohol a partir del vino de exudado obtenido de 15 dias de fermentacion despues de tres destilaciones se obtuvo del tratamiento de pasteurización (T1) un rendimiento de $58.4 \%$ y del tratamiento de no pasteurizacion (T2) un rendimineto de $76.09 \%$. En cuanto a la muestra de exudado de cacao dejada fermentar por 21 dias despues de dos destilaciones se obtuvo a partir de T1 un rendimiento de $48.28 \%$ y a partir de T2 un rendimiento $41.07 \%$. En ambos casos el alcohol obtenido corresponde a un alcohol etilico al $75 \%$.

En cuanto a Calidad del vino obtenido por tratamiento de las muestras enviadas al Laboratorio de Tecnología de Alimentos (LABAL-MIFIC), manifiestan lo descrito en la Tabla 4.

Tabla 4: Resultados de análisis de vino a partir de exudado de cacao

\begin{tabular}{|ccc|}
\hline \multicolumn{2}{|c|}{ Resultados de análisis de vino procedente de T1 } \\
\hline Pruebas de Calidad Realizadas & Resultados & Unidades \\
\hline Acidez Total & 2.16 & $\%$ \\
\hline Acidez Volátil & 0.36 & $\%$ \\
\hline Grado Alcohólico & 13.23 & $\%$ \\
\hline Azucares Reductores & 1.30 & $\%$ \\
\hline Metanol & 0.00 & $\mathrm{Mg} / 100 \mathrm{~mL}$ \\
\hline Resultados de análisis de vino procedente de T2 & \\
\hline Pruebas de Calidad Realizadas & Resultados & Unidades \\
\hline Acidez Total & 2.06 & $\%$ \\
\hline Acidez Volátil & 0.35 & $\%$ \\
\hline Grado Alcohólico & 12.61 & $\%$ \\
\hline Azucares Reductores & 1.32 & $\mathrm{Mg} / 100 \mathrm{~mL}$ \\
\hline Metanol & 0.00 & \\
\hline
\end{tabular}

Fuente: (LABAL L. d., 2015) 
Según él porcentaje de alcohol obtenido de $13.23 \%$ en T1 y $12.61 \%$ en T2, la cantidad de azucares reductores de $1.30 \%$ en el vino de T1 y 1.32 en T2 ambos vinos se clasifica en un vino seco, ya que para el vino seco el porcentaje de alcohol oscila en un rango de $8.5 \%$ a $14.5 \%$, con azúcares reductores menores a 5\%, según (Iris \& Ricardo, 2013), presentando una acidez volátil de 0.36 en un rango aceptable de consumo al encontrarse variando de 0.20 a 0.60 (Cazorla, Xirau Vayreda, \& Azorín Romero, 2011) y al no contener metanol lo hace apto para el consumo.

Con respecto a la aceptabilidad de los vinos los 67 panelistas apreciaron que el vino a partir del tratamiento de pasteurización T1 las diferencias encontradas al analizar los atributos de olor, color, apariencia, precio y presentación no presentaron diferencias significativas encontrándose en una hipótesis nula, en relación al sabor los encuestados percibieron un sabor de ácido a amargo con aceptación en el consumidor. En cuanto al vino procedente de T2 al igual que en T1 los atributos de olor, color, apariencia, precio y presentación no presentaron diferencias significativas pero se obtuvo más aceptabilidad en sabor al presentar un sabor de ácido a dulce.

Las pruebas realizadas al alcohol obtenido tanto del vino destilado con 15 días de fermentación como del vino destilado con 21 días de fermentación demostraron que era alcohol etílico al $75 \%$ de pureza, soluble en agua, con un $\mathrm{pH}$ acido de 5 y densidad de $0.79 \mathrm{gr} / \mathrm{ml}$, los que al momento de realizar la prueba de combustión no desprendían humo presentando una llama color amarillo y azul no luminosa, todo esto propio de un alcohol etílico al 75\%, según lo descrito por García, en el año 2001.

En cuanto a costos de produccion teniendo en cuenta los costos variable unitarios, costos indirectos de fabricacion y la produccion esprada, empleados en la investigacion tanto para la produccion del vino como del alcohol, se obtuvo para el vino a partir del tatamiento de pasteurizacion $C \$ 198.12$ y para el vino a partir de T2 de $C \$ 195.23$. El alcohol presento un costo toal de produccion de $\mathrm{C} \$ 157.58$ para T1 y C $\$ 124.47$ para T2.

\section{CONCLUSIONES}

La cooperativa cuenta con la disponibilidad de materia prima para el desarrollo de ambos procesos productivos, siendo el exudado extraído apto para su procesamiento, ya que el porcentaje de coliformes encontradas es bajo dentro del límite permitido, sin embargo se debe mejorar las condiciones del proceso para la obtención del mucilago la materia prima y garantizar su inocuidad.

La caracterización físico-química del exudado pasteurizado (T1) como no pasteurizado (T2), demuestran que esta materia prima es apta para su procesamiento y obtener productos como el vino y alcohol al presentar en su composición T1 el $9.91 \%$ de carbohidratos, $4.38 \%$ de alcohol, fibra $0 \%$ y T2 Carbohidratos 5,03 \%, alcohol 4,02\%, fibra $0 \%$ lo que es beneficioso para la fermentación del mosto. 
En cuanto al proceso productivo de los vinos es necesario cumplir con los 15 días de fermentación, ya que de lo contrario si se dan más días se daría la producción de ácido acético, llevando a una temperatura $75^{\circ} \mathrm{C}$ durante la pasteurización y activación de levadura a $39^{\circ} \mathrm{C}$.

No se evidenciaron diferencias significativas en los rendimientos obtenidos en el vino al aplicar ambos tratamientos, aprovechándose en T1 el $69.33 \%$ y de T2 el $70.31 \%$.

Con respecto al proceso de obtención de alcohol se debe cumplir los 15 días de fermentación para ambos tratamientos, ya que a partir de este se aprovecha para T1 el $58.4 \%$ y $76.09 \%$ para T2 después de las dos destilaciones, a diferencia de 21 días de fermentación obteniendo un rendimiento de $\mathrm{T} 1$ de $48.28 \%$ y $41.07 \%$ para $\mathrm{T} 2$ con tres destilaciones,

De acuerdo a características físico-químicas presentes en el vino obtenido del tratamiento de pasteurización con $13.23 \%$ de alcohol, pH de 3.12, 'Brix de 6.67 y características físicoquímicas del vino obtenido del tratamiento de no pasteurización con $12.61 \%$ de alcohol, pH 3.11 y ${ }^{\circ}$ Brix de 6.89, son considerados dentro de la clasificación de un vino seco.

En relación a los atributos de color, olor, apariencia y sabor ambos vinos fueron aceptados, sin embargo hubo más preferencia en sabor, por el vino proveniente del tratamiento de no pasteurización, reflejando que les gustaría obtener ambos vinos en presentaciones de $750 \mathrm{ml}$ con un precio $\mathrm{C} \$ 150$ por botella.

El alcohol obtenido según sus características físico- químicas se encuentra dentro de la clasificación de un alcohol etílico o etanol con un porcentaje de pureza del $75 \%$ y una combustión sin presencia de humo con una llama no tan luminosa.

Los costos totales de producción generados por la elaboración del vino a partir del tratamiento de pasteurización son de $\mathbf{C} \$ 198.12$ y para el vino del tratamiento de no pasteurización C\$195.23. Los Costos de producción generados por el alcohol del tratamiento pasteurizado son C\$157.58 y de C\$124.47 para alcohol de tratamiento no pasteurizado.

Se comprobó que se puede obtener vino y alcohol a partir del exudado de cacao con características comerciales, obteniendo mejores rendimientos y con menor costo de producción el vino y el alcohol obtenido del tratamiento de no pasteurización.

Valor que los productores puedan agregar, para hacerlo más rentable, productivo y beneficioso para la protección de nuestros suelos de montañas, para la ecología en general y a la vez, de mayor beneficio para los agricultores que los cultivan. Su siembra de ésta importante planta, beneficia la economía y la ecología del país. 
Alba V. Díaz Corrales; Yessin Mahela Calero Leiva; Zilgean Massiel Moreno Lazo

\section{REFERENCIAS}

Briones, D. A. (01 de 02 de 2015). MITRAB. Recuperado el 07 de 12 de 2015, de MITRAB: www.mitrab.gob.ni

Castellón, L. B. (16 de Noviembre de 2012). Chocolate Nica apuesta al exterior. La PrensaEconomia. Recuperado el 01 de Febrero de 2015, de Chocolate Nica apuesta al exterior. La Prensa-Economia: http://www.laprensa.com.ni/2012/11/16/economía/124026ambicioso-mussy/

Cazorla, D. J., Xirau Vayreda, D., \& Azorín Romero, R. (Enero de 2011). Enología. Recuperado el 17 de Agosto de 2015, de Enología: http://www.usc.es/caa/MetAnalisisStgo1/enologia.pdf

Clímaco Álvarez 1, E. P. (Octubre de 2002). Morfología de los frutos y características físicoquímicas del Mucílago del cacao de tres zonas del Estado Aragua. Recuperado el 19 de Marzo de 2015, de Morfología de los frutos y características físico-químicas del Mucílago del cacao de tres zonas del Estado Aragua: http://www.scielo.org.ve/scielo.php?pid=S0002-192X2002000400006\&script=sci_arttext

García, F. E. (2001). Requena, L. Vamos a Estudiar Química Orgánica:Propiedades de los alcoholes. Recuperado el 20 de Noviembre de 2014, de Requena, L. Vamos a Estudiar Química Orgánica:Propiedades de los alcoholes: http://www.salonhogar.net/quimica/nomenclatura_quimica/Propiedades_alcoholes.htm

Garzaro, D., Guerra Cedezo, F., \& Kalvatchev, Z. (Junio de 1998). Theobrama Cacao L: Un nuevo enfoqe para la nutricion y la salud. Recuperado el 25 de Agosto de 2012, de Theobrama Cacao $\mathrm{L}$ : Un nuevo enfoqe para la nutricion $y$ la salud: http://conectarural.org/sitio/sites/default/files/documentos/articulo6_2.pdf

Gueyer, E. B., \& Montealegre, E. (02 de Junio de 2003). Reglamento de la Ley de Equidad Fiscal con Reformas Incorporadas.Dirección general de ingresos. Recuperado el 01 de Diciembre de 2015, de Reglamento de la Ley de Equidad Fiscal con Reformas Incorporadas.Dirección general de ingresos: http://www.dgi.gob.ni/documentos/Decreto_46_2003_Reglamento_a_la_Ley_453,_Ley_ de_Equidad_Fiscal_con_sus_Reformas.pdf

INIFOM, I. N. (2011). Población: Esteli. Recuperado el 05 de Agosto de 2015, de Población: Esteli: https://webesteli.wordpress.com/asi-es-esteli/

Iris, \& R. D. (20 de Febrero de 2013). Enología y Enotécnia. Recuperado el 17 de Agosto de 2015, de Enología y Enotécnia: https://fundamentosdeenologia.wordpress.com/page/2/

LABAL, L. d. (Junio de 2015). Resultados de Analis de muestrasde exudado de cacao. Managua.

MINECO, M. d., CONACYT, C. N., MIFIC, M. d., SIC, S. d., \& MEIC, M. d. (10 de Septiembre de 2009). REGLAMENTO TÉCNICO CENTROAMERICANO. Recuperado el 07 de 
Alba V. Díaz Corrales; Yessin Mahela Calero Leiva; Zilgean Massiel Moreno Lazo

Septiembre de 2015, de REGLAMENTO TÉCNICO CENTROAMERICANO: http://digesto.asamblea.gob.ni/iunp/docspdf/gacetas/2009/9/g172.pdf

Munch, L., \& Angeles, E. (1996). Metodo y tecnicas de investigacion. Trillas.

Sandoval, D. M. (2013). IDENTIFICACIÓN Y CARACTERIZACIÓN DE ALCOHOLES Y FENOLES: QUIMICA ORGANICA II (1413). Recuperado el 25 de Noviembre de 2014, de IDENTIFICACIÓN Y CARACTERIZACIÓN DE ALCOHOLES Y FENOLES: QUIMICA ORGANICA II(1413): http://depa.fquim.unam.mx/amyd/archivero/Manualpracticas4247(2013-2)Sandoval_23404.pdf

Zeledón, E. Y., Arvizú Aráuz, S. G., \& González Urrutia, K. I. (Febrero de 2013). Produccion de postres y vinagre a partir del exudado de cacao en la cooperativa de servicios multiples "Ríos de agua viva, 21 de Junio" Rancho Grande, Matagalpa. Recuperado el 19 de Marzo de 2015, de Produccion de postres y vinagre a partir del exudado de cacao en la cooperativa de servicios multiples "Ríos de agua viva, 21 de Junio" Rancho Grande, Matagalpa: https://drive.google.com/file/d/0B3bbPp228aLcXdCdThUTTNXbUU/edit?pli=1

Zeledón, M. N. (2013). Formulacion y evaluacion de proyectos. Recuperado el 06 de Enero de 2015, de Formulacion y evaluacion de proyectos: mauricionavarrozeledon.files.worpress.com/2013/02/unidad-no-iv-estudioecononc3b3mico.pdf

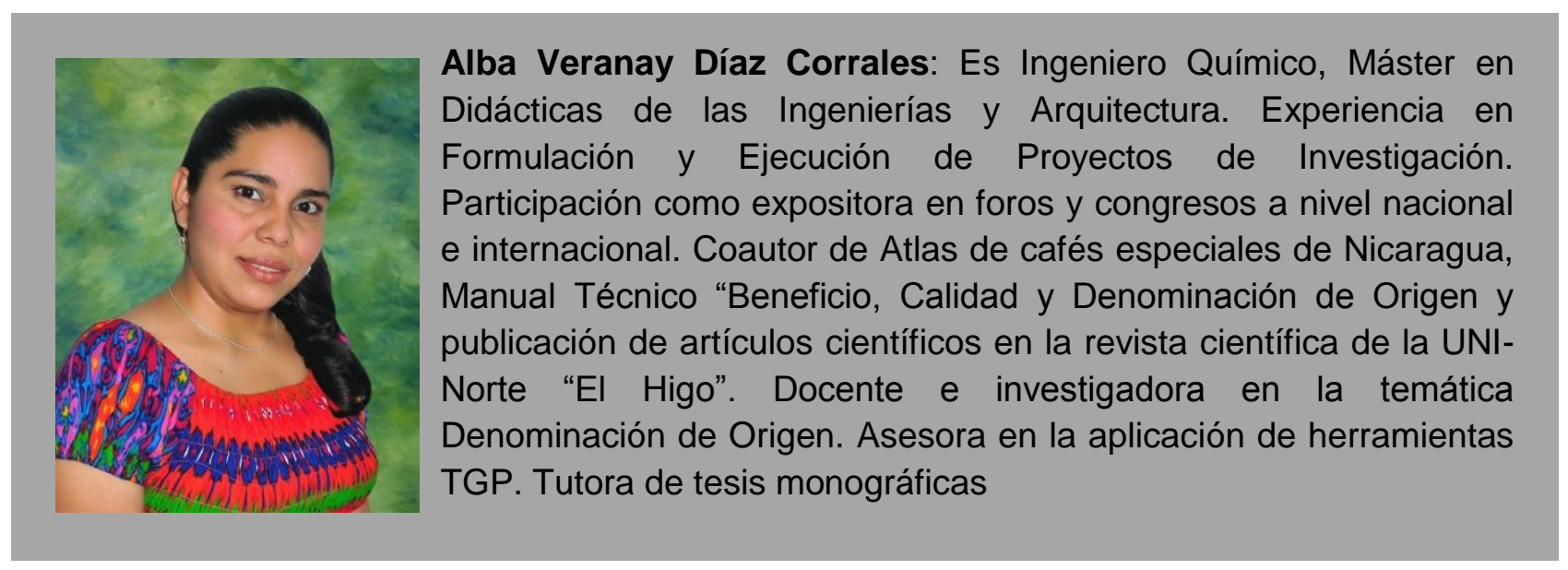


Alba V. Díaz Corrales; Yessin Mahela Calero Leiva; Zilgean Massiel Moreno Lazo
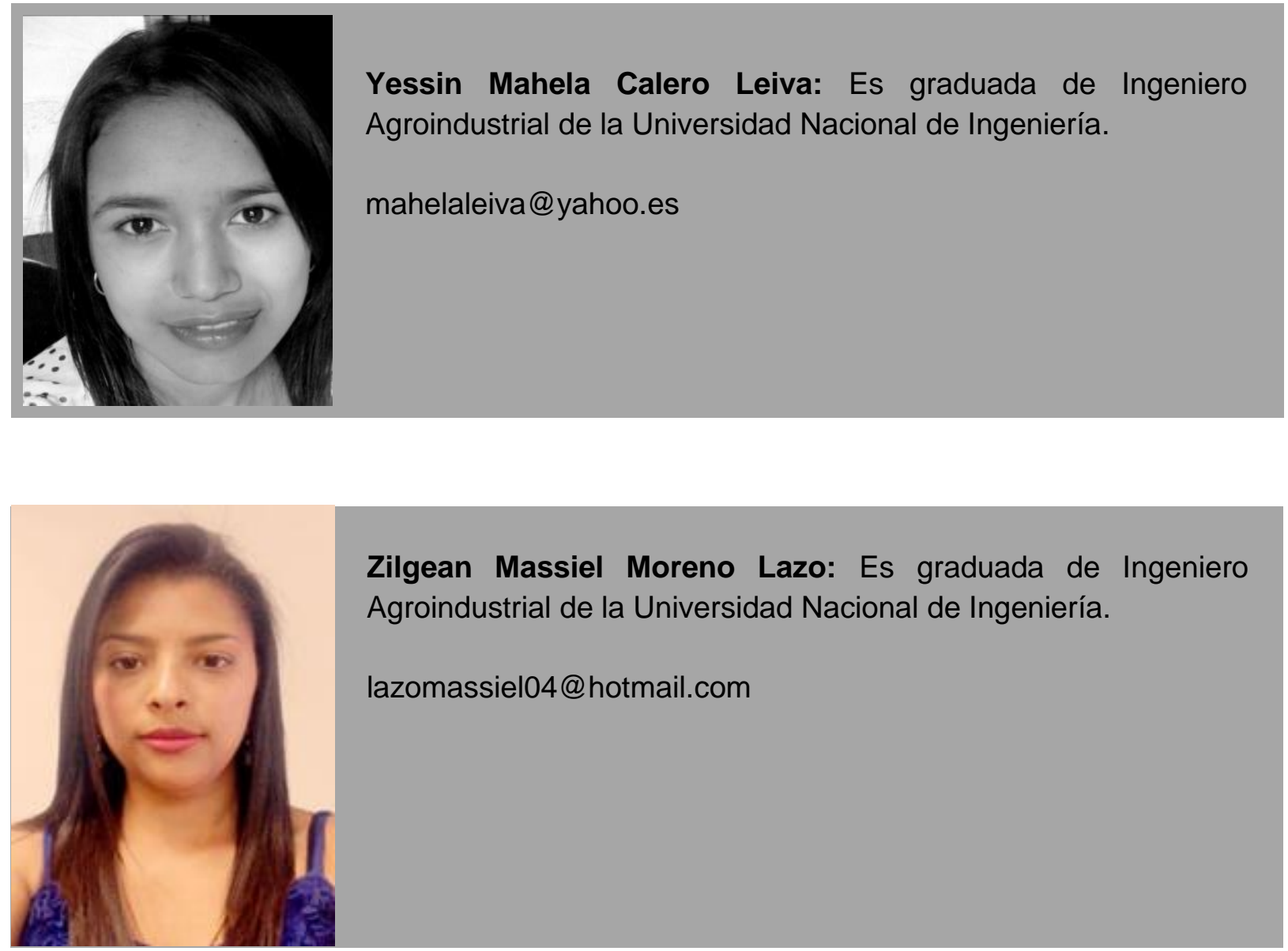